\title{
Unmanned Arial System for Medical Emergencies
}

\author{
Bharath $\mathrm{K} \mathrm{N}^{1}$, Yogesha B R ${ }^{2}$, Karthik N S ${ }^{3}$, Anusha G T ${ }^{4}$, Ramya M R \\ Department of $E \& C$, Assistant Professor ${ }^{l}$, Students ${ }^{2,3,4,5}$ GMIT, Bharathinagara, Mandya ${ }^{1,2,3,4,5}$ \\ Email: knb1990@gmail.com ${ }^{1}$, parthapuru96@gmail.com ${ }^{2}$
}

\begin{abstract}
In the case of a medical emergency such as an out of-hospital cardiac arrest, the chances of a person's survival decrease rapidly if they are not attended to immediately. Modern hospitals well equipped to deal with such a situation however, an ambulance may get stuck in traffic and it may take responders time to reach locations deep within a building. Each year between 180,000 and 400,000 people die due to cardiac arrest. However, it is possible to reduce this number. Unmanned Aerial Vehicles (UAV) has regularly been used for remote sensing and aerial imagery collection, but the technology exists to allow the use of drones to respond to medical emergencies. To ensure that drones can reach the victim and provide medical aid, a framework for reacting to emergency circumstances, is required. This paper proposes a system-of systems-based framework that is capable of responding to one of the most acute medical emergencies, a heart attack. The proposed architecture consists that work in coordination to ensure that the drone can achieve safe flight and provide medical aid. An autonomous command and control system is discussed. This system also allows manual control from a remote location.
\end{abstract}

Keywords: Electronic Speed Controller (ESC), APM 2.5 Flight Controller, BLDC Motor.

\section{INTRODUCTION}

In case of a medical emergency such as outof-hospital cardiac arrest, which is prevalent in the United States, the chances of a person's survival decreases very fast if not attended to immediately. Modern hospitals are well equipped to deal with such situations, however the ambulance could get stuck in traffic further delaying the chance of survival. Each year between 180,000 and 400,000 people die due to cardiac arrest1.2. One of the important components of successful cardiac arrest treatment is emergency medical services (EMS) response time. Unmanned Aerial Vehicles (UAV) has regularly been used for remote sensing and aerial imagery collection, but there are new opportunities to use drones for medical emergencies3. The purpose of this study was to develop a geographic approach to the placement of a robotic arm on medical drones, equipped with an automated external defibrillator, designed to minimize travel time to victims of out-of-hospital cardiac arrest.

\section{LITERATURE SURVEY:}

The quad copter will reach the accident scenario by tracking the location using GPS. The real time condition of the patient details will be send to the hospital and to the ambulance using GSM technology [1]. Focused on the Asphyxiation patients and their urgent need of oxygen supply. The drone could support a patient who is about an hour away from ambulance support. To get the oxygen support this can last up to 3 and half hour [2]. This paper proposes a fully automatic charging station which operates wirelessly. The station also allows for imprecise landing of the UAV on the platform, which is often the case for practical systems [3].

Ardu-pilot Mega (APM ver.2.6) is the Flight Controller used in this project; this autopilot can also control fixed-wing aircraft, multi-rotor helicopters, as well as traditional helicopters. It is an autopilot capable of autonomous flight stabilization, way-point based navigation and two way telemetry with zegbee wireless [4]. While engaged in the Global War on Terror, our soldiers have discovered the need at the small unit level for a non-line of sight, man portable, lethal, unmanned device that could be used to gain entry into a building by blowing the door, kill enemy personnel or disable soft skinned vehicles without a soldier ever exposing himself to the enemy and while minimizing collateral damage [5].

While engaged in the Global War on Terror,our soldiers have discovered the need at the small unit level for a non-line of sight, man portable, lethal,unmanned device that could be used to gain entry into a building by blowing the door, kill enemy personnel or disable soft skinned vehicles without a 


\section{International Journal of Research in Advent Technology, Vol.7, No.5, May 2019 E-ISSN: 2321-9637 \\ Available online at www.ijrat.org}

soldier ever exposing himself to the enemy and while minimizing collateral damage [6]. As highly maneuverable drones are available at the low price, the threats that might be caused by the drone attacks has been increased. Recent object detectors have been dramatically improved in accuracy by using convolutional neural networks, and these can be utilized to identify hostile drones. In this paper, we examine state-of-the-arts convolutional object detectors for a real-time drone detection and tracking system using a Pan-Tilt-Zoom (PTZ) camera. In the drone detection and tracking system, an object detector is used to identify whether an image from the PTZ camera contains a drone, and our system generates PTZ actions to track the detected drone. To detect small size drones in real-time, an appropriate object detector should be selected. This paper compares six convolutional object detectors in the accuracy and speed [7]. Localization system for objects tagged with UHF-RFID passive tags, where the reader is attached to a drone. The system implements the phase-based SARFID technique to locate static tags with respect to a UHFRFID reader attached to a commercial drone. The reader antenna trajectory is achieved through a Global Positioning System. The bi dimensional tag position is estimated with centimeter order accuracy. Only one reader antenna is required, without any reference tag [8].
An innovative and first system in the world for the drone to conduct the assigned self emission in a fully autonomous manner. In this system, the users can select a number of photo-templates on the APP and then the drone will autonomously fly to required positions to take the photos. In the selfie-photo templates, human stays in varied position, viewing angles, and distances to the drone inside the images. Human selects a preferable template, and then the drone will fly and shoot one photo that matches all parameters to the template without any manual control. The selfie drone system transforms the selected photo template into parameters which are subsequently used to perform the image-based visual servoing based control to enable the drone to fly to necessary position to shoot the requested selfie-photo in a fully autonomous manner. The innovative drone selfie system has been proven effective in a number of experiments [9].

\section{PROPOSED METHODOLOGY}

Fig. 1 Show block diagram of our manual drone. The working principle of the system is depicted in Fig.1 There are two techniques to identify cardiac arrest either by mobile phone application or bodyattached sensor.

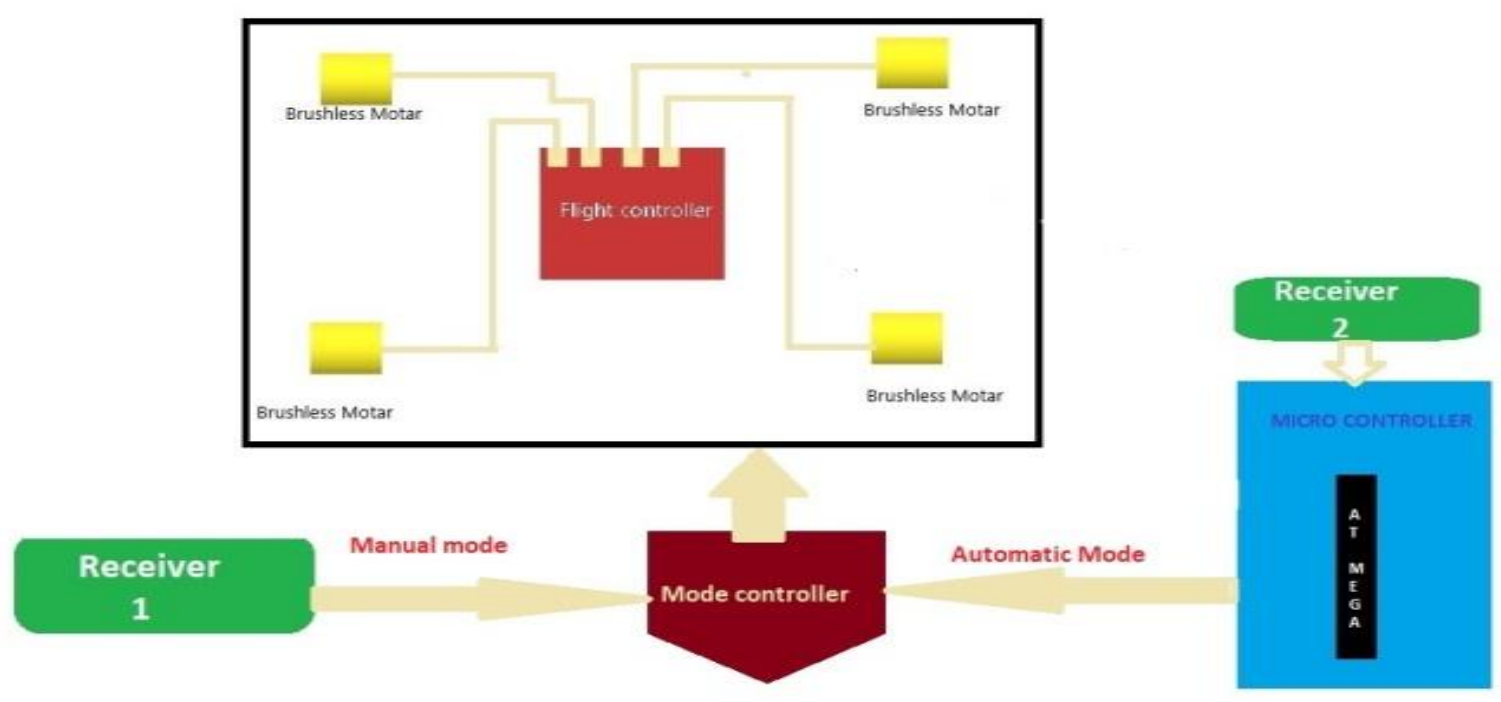

Fig (1) Methodology of drone

These techniques will send a message to the drone dispatch system using Global Positioning System (GPS). The coordinates received from the
GPS signal will be converted to street map using GIS parser. The information management integrates the GPS location and emergency database information. 


\section{International Journal of Research in Advent Technology, Vol.7, No.5, May 2019 \\ E-ISSN: 2321-9637 \\ Available online at www.ijrat.org}

In emergency database, basic information regarding the person is maintained such as personal contacts, height, weight, and blood. Proximity analysis is calculated with the help of GPS coordinates of the victim to deploy drone to the site. The drone dispatch center receives the GPS information from the victim, the system finds shortest path and incorporates map into the drone using GIS techniques. The drone reaches the destination and handovers the AED kit to the person.

There are several issues to be considered while preprocessing the depth images. First, the images captured by the camera might have wrong pixel in certain areas. This problem can be solved by using neighborhood interpolation techniques. It assigns a value to the invalid pixel based on the values of its neighborhood pixels15. Second, the images captured may contain optical noise which might produce irregular edges. This can be resolved by bilateral filtering of the image.

\section{COMPONENTS REQUIRED}

\section{Hardware tools:}

\subsection{BLDC Motors}

A brushless DC motor (also known as a BLDC motor or BL motor) is an electronically commuted DC motor which does not have brushes. The controller provides pulses of current to the motor windings which control the speed and torque of the synchronous motor.

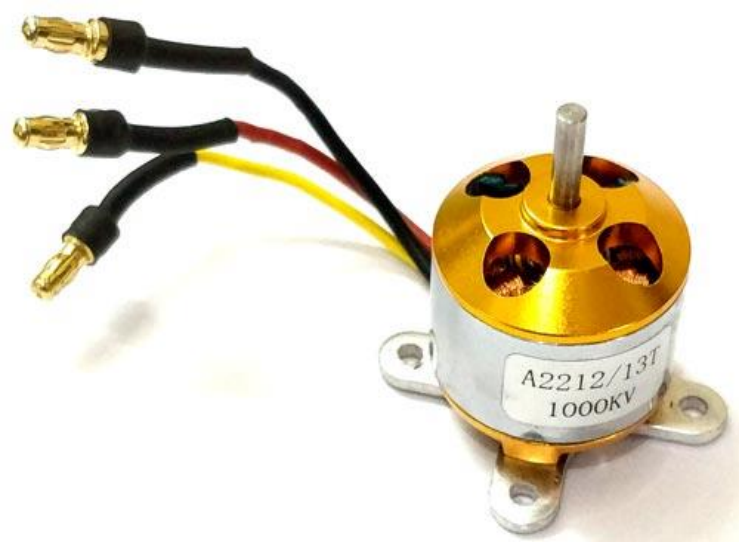

Fig (2) BLDC Motor

\subsection{F450 Frame}

This is a $450 \mathrm{~mm}$ size quadcopter frame that is made from plastic and fibreglass. It includes an integrated PDB. This F450 flaming wheel compatible frame is ideal for new users who would like to build thier first quadcopter as this is a very affordable and strong frame that can handle crashes. Similarly this frame can be used by more advanced users who would like a simple lightweight platform to try more aggressive stunt flying without the worry of breaking an expensive quadcopter frame.

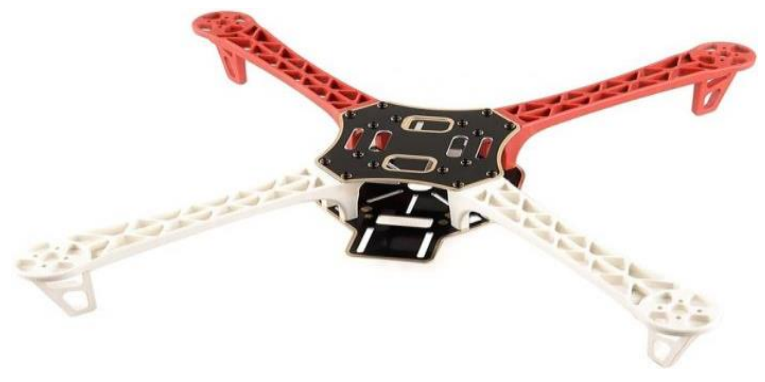

Fig (3) F450 Frame

\subsection{Electronic Speed Controllers}

An electronic speed control or ESC is an electronic circuit that controls and regulates the speed of an electric motor. It may also provide reversing of the motor and dynamic braking. Miniature electronic speed controls are used in electrically powered radio controlled mode

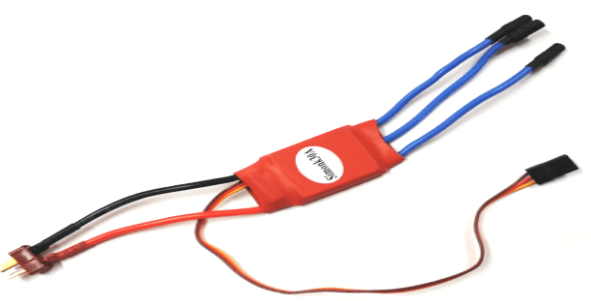

Fig (4) ESC

\subsection{LIPOO Battery}

A lithium polymer battery, or more correctly lithiumion polymer battery (abbreviated as Li-Po, LIP, Lipoly, lithium-poly and others), is a rechargeable battery of lithium-ion technology using a polymer electrolyte instead of a liquid electrolyte. 


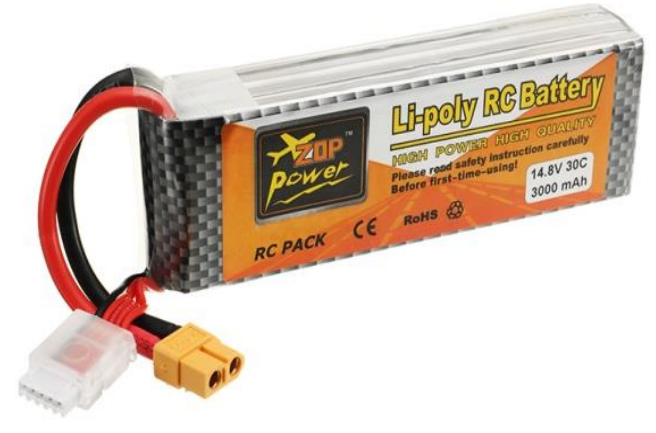

Fig (5) Li-poly battery

\subsection{Flight Control System (KK2.1)}

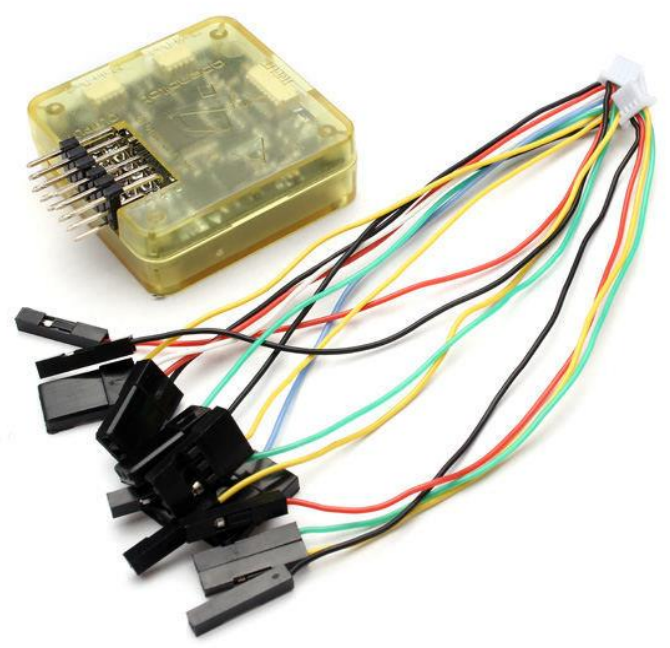

Fig (6) Flight controller

A flight controller (FC) is a small circuit board of varying complexity. Its function is to direct the RPM of each motor in response to input. A command from the pilot for the multi-rotor to move forward is fed into the flight controller, which determines how to manipulate the motors accordingly.

\subsection{Fly sky fs-i6 radio}

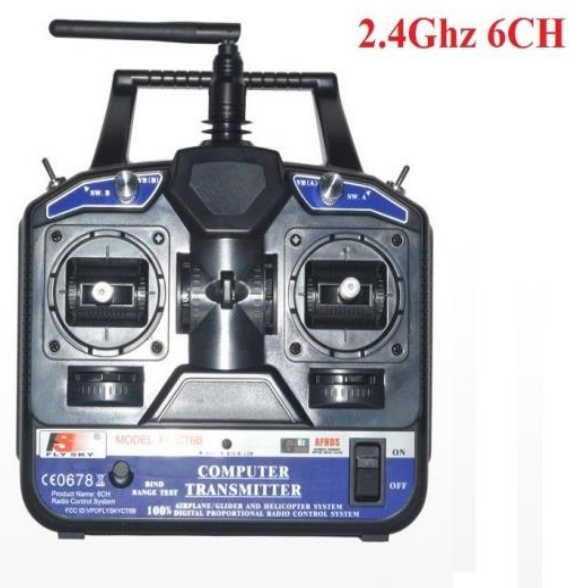

Fig (7) Fly sky fs-i6 radio

\subsection{GPS system}

The Global Positioning System (GPS) is made up of satellites, ground stations, and receivers. GPS is a system. Once the receiver calculates its distance from four or more satellites, it knows exactly where you are!

\subsection{Carry Bag for medicines}

It is used to carry the medicine.

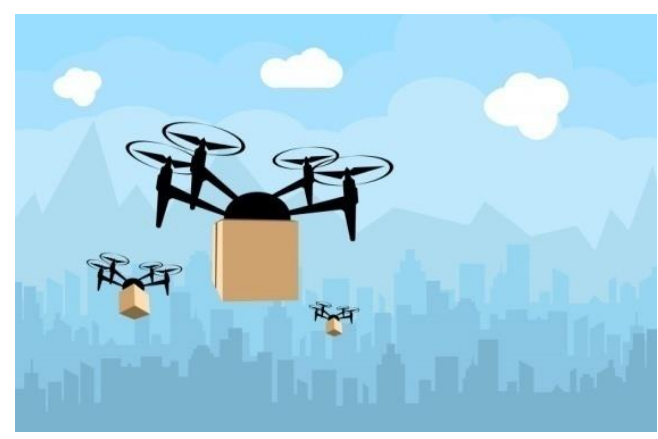

Fig (8) Drone carrying a medicine

\section{Software tool:}

\subsection{LibrePilot}

Free software unmanned aerial vehicle project for model aircraft aimed at supporting both multi-rotor craft as well as fixed-wing aircraft. Initially founded by David Anker's, Angus Peart and Vassals Varveropoulos in late 2009, under the name Open 


\section{International Journal of Research in Advent Technology, Vol.7, No.5, May 2019 E-ISSN: 2321-9637 \\ Available online at www.ijrat.org}

Pilot, it was conceived as both a learning tool and to address areas the developers perceived were lacking in other small UAV platforms. In July 2015 Open Pilot, was forced to create LibrePilot.

The Open Pilot open source autopilot software could be combined with hardware such as an inertial navigation system board, a main control board, a GPS receiver, and a $2.4 \mathrm{GHz}$ serial communications link with the ground station. ${ }^{[4]}$ The Open Pilot software is released under the GPL version 3 licenses.

\section{RESULT AND APPLICATIONS}

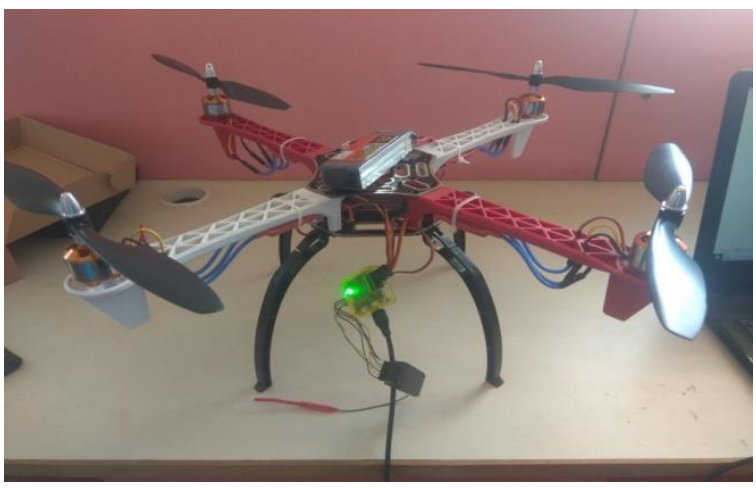

- Medical Emergency

- Aerial photography

- Disaster management

- Entertainment

6. CONCLUSION

Unmanned Aerial Vehicles (UAV) has regularly been used for both manual and automatic, but there are new opportunities to use drones for medical emergencies. To ensSure that drones can reach the person and provide medical aid, a completely practical framework equipped for reacting to these circumstances, is required. This paper has proposed an interconnected system of systems framework capable of responding to a medical emergency. The interconnected drone system has been explained briefly, which can monitor and provide emergency response.

\section{REFERENCES}

[1] Dr.J.Prem Kumar, "Quad copter based technology for an emergency health care", IEEE paper -2011

[2] Syed Ali shahbaz, "Ambulance Drone support system (ADSS)”, International Journal of
Research in Communication Engineering, paper-2015

[3] "Automatic Wireless Drone Charging Station". Chung Hoon Choi, Hyeon Jun Jang, Seong Gyu Lim, Hyun Chul Lim, Sung Ho Cho, Igor Gaponov, IEEE 2016.

[4] "Fully Autonomous UAV", K.R. Jansi, Anup Venkatesh G, Sumanth P, IEEE 2017.

[5] "Small unit unmanned weapon system for today's army" CDT Julia D. Carier, CDT Earnest D. Smith, CDT Andrew M. Wade, CDT Paul S. Walker, LTC Michael J.IEEE 2015.

[6] "Innovative Drone Selfie System and Implementation", Chyi-Yeu Lin and Chung-Yi Liang Department of Mechanical Engineering. National Taiwan University of Science and Technology, 2017.

[7] "Comparison of Convolutional Object Detectors for Real-time Drone Tracking Using a PTZ Camera" by Jihun Park*, Dae Hoe Kim, Young Sook Shin, Sang-ho Lee, Agency of Defense Development, Daejeon, Korea.2017

[8] "Drone-based UHF-RFID Tag Localization" A. Buffi, P. Nepa. Department of Information Engineering, University of Pisa, Italy.2014

[9] Rahul Gomes. Department of Computer Science, North Dakota State University.IEEE 2017.

[10] Karanam Ravichandran Dayananda Department of Computer Science, North Dakota State University.IEEE 2017 